\title{
¿QUÉ INFORMACIÓN SOBRE LAS TASAS DE INTERÉS SPOT FUTURAS CONTIENE LA ESTRUCTURA TEMPORAL DE TASASDE INTERÉS EN MÉXICO?
}

\author{
Sara Gabriela Castellanos y Eduardo Camero
}

Marzo de 2002

Documento de Investigación No. 2002-3

Dirección General de Investigación Económica

BANCO DE MÉXICO

\footnotetext{
* Ambos autores agradecen los comentarios de los participantes en los seminarios de la DEE del Banco de México, El Colegio de México y el Centro de Investigación y Docencia Económica, la V Reunión de LACEA y la V Reunión de la Red de Investigadores de Banca Central de las Américas. Un agradecimiento especial corresponde a Alejandro Werner, Daniel Garcés y Gerardo Esquivel. Los juicios emitidos son responsabilidad única de los autores. Las opiniones expresadas en este documento no necesariamente reflejan las del Banco de México.
} 


\title{
¿QUÉ INFORMACIÓN SOBRE LAS TASAS DE INTERÉS SPOT FUTURAS CONTIENE LA ESTRUCTURA TEMPORAL DE TASASDE INTERÉS EN MÉXICO?
}

\author{
Sara Gabriela Castellanos y Eduardo Camero
}

Marzo de 2002

Documento de Investigación No. 2002-3

Dirección General de Investigación Económica

BANCO DE MÉXICO

\begin{abstract}
Resumen
En este documento se examina la relación que existe entre las tasas de interés spot y la estructura temporal de tasas de interés $(E T T I)$. El análisis se realiza mediante algunas pruebas derivadas de la hipótesis de expectativas racionales. Los resultados sugieren que la ETTI contiene cierta información sobre la dirección de los movimientos futuros de las tasas de interés spot tanto de corto como de largo plazo. Ello, no obstante el rechazo que se obtiene de la hipótesis de expectativas racionales en las pruebas realizadas. Las estimaciones con modelos GARCH y GARCH-M que se llevan a cabo parecen indicar que este resultado se relaciona con la existencia de variaciones en las primas de riesgo asociadas a las tasas de interés.
\end{abstract}




\section{Introducción}

Existe una extensa literatura empírica en la cual se busca documentar si la estructura temporal de las tasas de interés (ETTI), o bien, el diferencial entre las tasas de interés de largo y de corto plazo, pueden predecir en la dirección correcta los cambios de las tasas de interés de corto plazo. Según la hipótesis de expectativas (HE) preferida en la teoría económica para interpretar la relación entre estas variables, cuando la tasa de interés de largo plazo aumenta con respecto a la tasa de interés de corto plazo, las tasas de interés de corto plazo futuras tienden a incrementarse. Esto se debe a que según la $\mathrm{HE}$, las tasas de interés de largo plazo corresponden a promedios ponderados de las tasas de interés de corto plazo presentes y futuras. Por tanto, acorde con este marco de análisis, el incremento de las tasas de interés de largo plazo con respecto a las de corto plazo se debe a una expectativa de mayores tasas de interés de corto plazo en el futuro. Esto es así, sujeto a que el mercado realice una predicción correcta en promedio.

El propósito de este documento es analizar la relación entre la ETTI y las tasas de interés de largo y de corto plazo. Para tal fin se utiliza como marco de análisis la HE. Además de contribuir a documentar la evidencia empírica sobre esta relación, lo cual se considera un ejercicio relevante para toda ciencia, esta investigación es útil por razones más prácticas. Una de ellas es que, en general, el seguimiento de h ETTI es uno de los varios mecanismos disponibles para diversos agentes económicos participantes en los mercados financieros para recabar e interpretar información sobre las expectativas de movimientos de las tasas de interés que, a su vez, pueden utilizarse para tomar decisiones de consumo o de inversión. Por tanto, comprender mejor las ventajas y desventajas de este mecanismo con respecto a otros alternativos -encuestas directas o modelos estadísticos, por ejemplo- resulta de importancia. Entre los agentes económicos que participan en los mercados financieros, destacan particularmente las autoridades monetarias, debido a la influencia que sus acciones de política pueden tener sobre las tasas de interés de diferentes plazos. ${ }^{1}$ Por lo anterior, se considera de especial relevancia determinar si las tasas de mayor plazo o las tasas de corto plazo futuras

\footnotetext{
${ }^{1}$ Por ejemplo, Castellanos (2000) y Díaz de León y Greenham (2000) encuentran que los efectos de la política monetaria del Banco de México, instrumentada mediante el anuncio del saldo objetivo para las cuentas de los bancos comerciales en el propio banco central (denominado comúnmente como "el corto"), tiene precisamente un mayor efecto sobre las las tasas de interés con vencimientos a 1, 28 y 91 días que sobre aquéllas con vencimientos a 182 y 364 días.
} 
reflejan eventualmente los efectos de la política monetaria o de otras perturbaciones sobre las tasas de interés de corto plazo.

Siendo el objetivo de esta investigación documentar la relación entre la ETTI y las tasas de interés spot para México y no la comprobación exhaustiva de la HE, se seleccionan algunas de las pruebas más importantes. Conforme los resultados de estas pruebas sugieren el rechazo de la hipótesis de expectativas -que es el resultado más común de los estudios empíricos con datos tanto de México como de otros países- se añaden pruebas adicionales que ayudan a comprender por qué se da tal rechazo. El análisis emplea datos de las tasas de interés de los CETES a 28, 91, 182 y 364 días en el periodo de febrero de 1986 a diciembre de 2000. Los resultados sugieren que aunque la ETTI contiene información sobre las tasas de interés spot futuras, los cambios de las tasas de interés difieren de los predichos por la hipótesis de expectativas. Los cambios futuros de las tasas de interés de corto plazo son menores que los sugeridos por el diferencial entre las tasas de interés de corto y largo plazo. En la investigación se exploran dos posibles explicaciones de este resultado. Una de ellas se basa en la idea de que es imposible predecir los cambios esperados de las tasas de interés utilizando información contemporánea. La otra posible explicación, que parte de la posibilidad de predecir los cambios esperados en las tasas de interés, se basa en la existencia de una prima de riesgo que sea variable a través del tiempo.

Las estimaciones realizadas con los rezagos de las propias tasas de interés, al mostrar que tienen cierto poder predictivo en cuanto a sus cambios a futuro, permiten descartar la primera explicación. Por otra parte, se encuentra evidencia en favor de la segunda explicación al examinarse la volatilidad del diferencial entre las tasas de interés forward y spot, la cual puede ser atribuida a cambios en las primas de riesgo. Esto se verifica más formalmente mediante el uso de modelos de autocorrelación y heteroscedasticidad condicional generalizada. $^{2}$ Dichos ejercicios parecen confirmar el poder predictivo de los rezagos del diferencial de las tasas y del premio al riesgo sobre las primas de riesgo, aún controlando por la posibilidad de que éstas fluctúen. Por tanto, los resultados obtenidos parecen relacionarse con la existencia de una prima de riesgo fluctuante a través del tiempo.

El resto del documento se divide en 5 secciones. En la sección 2 se revisan los estudios previos realizados para México sobre el tema. En la sección 3 se describen las pruebas 
empleadas y los datos. En la sección 4 se presentan las estimaciones realizadas. Finalmente, la sección 5 contiene las conclusiones principales.

\section{Estudios Previos}

El tema sobre el poder de predicción de la ETTI acerca del comportamiento de las tasas de interés spot futuras es uno de los aspectos más importantes de la relación entre los mercados spot, los mercados futuros y los diferenciales de tasas. Esta cuestión ha sido abordada vastamente en la literatura teórica y empírica. ${ }^{3}$ Para comprender los resultados de los estudios sobre la ETTI y las tasas de interés que emplean el marco de análisis de la HE, es útil iniciar con la presentación de dos versiones de dicha hipótesis. La hipótesis pura de expectativas de la ETTI plantea que las tasas de interés deben comportarse de manera tal que den a un inversionista el mismo rendimiento esperado con independencia del plazo de su estrategia de inversión. A su vez, la hipótesis de expectativas sugiere la proposición más débil de que la diferencia entre los rendimientos esperados obtenidos de estrategias de inversión con diferentes plazos debe permanecer constante en el tiempo. Esto implica que las tasas de largo plazo dependan de las tasas de interés de corto plazo corrientes y esperadas y que, a su vez, la pendiente de la curva de rendimientos, el diferencial entre las tasas de interés de largo y corto plazo, refleja el pronóstico del mercado acerca de los cambios en las tasas de interés. Obsérvese que ambas versiones hablan de rendimientos esperados, lo cual implica que probar la hipótesis de expectativas requiere hacer algún supuesto sobre cómo forman sus expectativas los agentes económicos. ${ }^{4}$ Los supuestos más usuales sobre las expectativas de los agentes son que éstos tienen expectativas racionales, de manera que sus pronósticos utilizan toda la información disponible y no producen errores sistemáticos (es decir, que tienen media cero y errores tipo ruido blanco), o bien, que éstos tienen previsión perfecta, de manera que sus pronósticos siempre son correctos.

\footnotetext{
${ }^{2}$ Generalized Autorregresive Conditional Heteroskedastic Models

${ }^{3}$ McCulloch y Schiller [31] presenta una revisión muy completa de este tema.

${ }^{4}$ En realidad, se reconoce que una de las dificultades más importantes para interpretar las pruebas de la HE es precisamente, que muchas de ellas son pruebas conjuntas de HE con algún proceso de formación de expectativas. Así, el rechazo de la prueba puede deberse a que no se cumple la hipótesis de expectativas o a que los agentes no forman sus expectativas de la manera supuesta.
} 
Campbell y Schiller (1991) observan, con respecto a estudios realizados con datos para los Estados Unidos, que "-la- literatura sobre la ETTI contiene una variedad sorprendente de respuestas a estas dos preguntas... -1) Si el diferencial entre las tasas de largo plazo y corto plazo predice cambios futuros en las tasas de interés, y 2) De ser así, si este poder predictivo del diferencial de tasas es congruente con la teoría de expectativas sobre la ETTI, la cual es, precisamente, la explicación más prevaleciente sobre la ETTI.-... Casi todos los estudios rechazan estadísticamente la hipótesis de expectativas de la ETTI, pero algunos estudios sugieren que el diferencial de tasas sí predice cambios en las tasas de interés de la manera amplia que uno esperaría si es verdad la hipótesis de expectativas, mientras que otros estudios obtienen una conclusión opuesta. Diferentes estudios usan diferentes métodos econométricos, prueban implicaciones diferentes de la teoría de expectativas y analizan tasas de interés con diferentes vencimientos." 5

Esta observación también se cumple en buena medida para los estudios previos efectuados con datos de México. Galindo (1995), Sod (1997) y González, et al (1999) realizan algunos de estos estudios en los que se ha abordado la relación entre la estructura temporal de las tasas de interés y las tasas de interés spot futuras, en el contexto de la validez de la hipótesis de expectativas racionales. Por tanto, resulta conveniente discutir brevemente las pruebas de la hipótesis de expectativas racionales mediante una referencia a las que se han empleado en dichos estudios.

Galindo (1995), mediante un análisis del orden de cointegración de las series de tasas de interés de los CETES con vencimientos a 1 y 3 meses en el periodo de 1990 a 1995 y el uso la metodología del VAR4 aplicada según Campbell y Schiller (1987), obtiene resultados en los que se rechaza la HE con expectativas racionales, ${ }^{6}$ pero son indicativos de que existe una relación estable de largo plazo entre las tasas de interés de corto y largo plazos. Su estudio encuentra evidencia de que los valores corrientes y pasados del diferencial de las tasas de

\footnotetext{
${ }^{5}$ Traducción de los autores.

${ }^{6}$ La metodología del VAR4 aplicada al análisis de las expectativas racionales sugiere tres pruebas básicas: 1) El diferencial de las tasas de interés debe causar, en el sentido de Granger, los cambios futuros de las tasas de interés de corto plazo. 2) La razón de varianzas entre el valor real del diferencial de tasas y el valor teórico de la estructura de las tasas de interés, según la hipótesis de expectativas racionales, deberá ser igual a 1, lo mismo que el coeficiente de correlación entre ambos. 3) El no rechazo de la prueba de Wald de las restricciones que validan la representación de $\mathrm{Z}=\left[\mathrm{R}_{\mathrm{t}}^{\mathrm{n}}-\mathrm{R}_{\mathrm{t}}^{\mathrm{m}}, \Delta \mathrm{R}_{\mathrm{t}}^{\mathrm{m}}\right]$ a través de un sistema de vectores autorregresivos de orden $\mathrm{p}$. Galindo (1995) y Campbell y Schiller (1987) desarrollan en mayor detalle la teoría que sustenta estas pruebas.
} 
interés y los de cambios en las tasas de corto plazo contienen información relevante para predecir cambios futuros en las tasas de interés.

Sod (1997) explora varias formas de la versión de previsión perfecta de la HE siguiendo a Mankiw (1986) y a Mankiw y Miron (1986) y el enfoque de VARs de Campbell y Schiller (1987) y (1991) con tasas de CETES con vencimientos a 1 y a 3 meses de 1982 a 1995 . Los resultados de estas pruebas permiten rechazar la HE pura, en general. Pero en una de las pruebas realizadas, que busca verificar si es imposible predecir la prima de riesgo a partir de su propia historia, los resultados sugieren que la hipótesis de expectativas tiene cierta validez. Estos resultados incentivan a realizar un análisis mediante procesos ARCH-M y GARCH-M. Las estimaciones correspondientes sugieren que los diferenciales de las tasas de interés incorporan una prima de riesgo fluctuante. Sod plantea que este resultado es una posible explicación para el rechazo de la hipótesis de expectativas.

En contraste con los hallazgos de esos dos estudios, los resultados de González et al (1999) aportan evidencia en favor de la HE pura con expectativas racionales. González et al (1999) realizan dos pruebas con datos de CETES a plazos de 1, 3 y 6 meses para el periodo de 1991 a 1996. En la primera de ellas, basada en Schiller, Campbell y Schoenholtz (1983), se obtiene que el coeficiente de la pendiente en la regresión entre el cambio inmediato de la tasa spot y la diferencia presente entre las tasas forward y spot es significativo. Esto se aprecia tanto en el periodo completo como en las dos muestras que consideran, 1991-1994 y 1995-1996, respectivamente. Sin embargo, en el estudio no se verifica que el coeficiente sea igual a 1 , lo cual también es requerido en la prueba. ${ }^{7}$ En la segunda prueba, basada en Campbell y Schiller (1991) y Engsted (1996), se estima el coeficiente de la pendiente en la regresión entre el cambio inmediato en las tasas de interés y el diferencial presente entre las tasas de largo y corto plazo, el cual resulta significativo. No obstante, algunos autores consideran que esta segunda prueba no se deriva exactamente de la teoría porque las tasas de interés pueden no cambiar en el periodo siguiente, excepto cuando la tasa de interés de largo plazo tiene vencimiento a 2 meses y la de corto plazo a 1 mes y, en consecuencia, se dice que ese resultado sólamente es sugestivo. ${ }^{8}$

\footnotetext{
${ }^{7}$ Fama (1984).

${ }^{8}$ Para una discusión más detallada, véase Mankiw (1986).
} 
Esta revisión de los estudios para México permite concluir que en México, lo mismo que para otros países, la variedad de pruebas y de resultados sobre la HE hacen posible tanto aceptar como rechazar dicha hipótesis. En la siguiente sección, al describir las pruebas seleccionadas para este estudio, se explica con mayor detalle porqué algunas arrojan resultados que algunos autores han interpretado como evidencia en favor de la HE y en qué medida lo son.

\section{Datos y Metodología}

En esta sección se describen, primeramente, las tasas de interés que se emplean para construir las variables requeridas para realizar el presente análisis, así como sus propiedades de estacionariedad y de causalidad de Granger. Luego, se explican las dos principales pruebas empíricas de la HE seleccionadas.

Las tasas de interés que se utilizan para este estudio provienen de los resultados de las subastas primarias semanales de los Certificados de la Tesorería (CETES) de febrero de 1986 a agosto de 2000. Los CETES son bonos con cupón cero emitidos por el Gobierno Federal de México ${ }^{9}$ a plazos de 28, 91, 182, 364 y 728 días. Sin embargo, por el reducido número de observaciones en el periodo de la muestra de las emisiones a 728 días, estos bonos no se incluyen en el análisis. En la Gráfica 1 se pueden apreciar las observaciones con periodicidad mensual. Destacan algunos saltos de las tasas de los CETES a 182 y 364 días debidos a los periodos en que dichos títulos no fueron ofrecidos por el Gobierno Federal. Se decidió excluir esos periodos de las estimaciones en razón del efecto adverso que podrían ejercer sobre ta estabilidad de los coeficientes, inclusive al considerar diferentes particiones de los datos. A partir de estas tasas de interés se construyen las variables tanto de cambios de las tasas de corto o de largo plazo, como de los diferenciales entre tasas de corto y largo plazo, entre tasas forward y spot, y mediciones del premio al riesgo requeridas en las pruebas de la HE.

\footnotetext{
${ }^{9}$ Esto implica que únicamente están expuestos a riesgo soberano.
} 


\section{Gráfica 1}

Tasas de Interés de los CETES, Febrero 1986-Agosto 2000

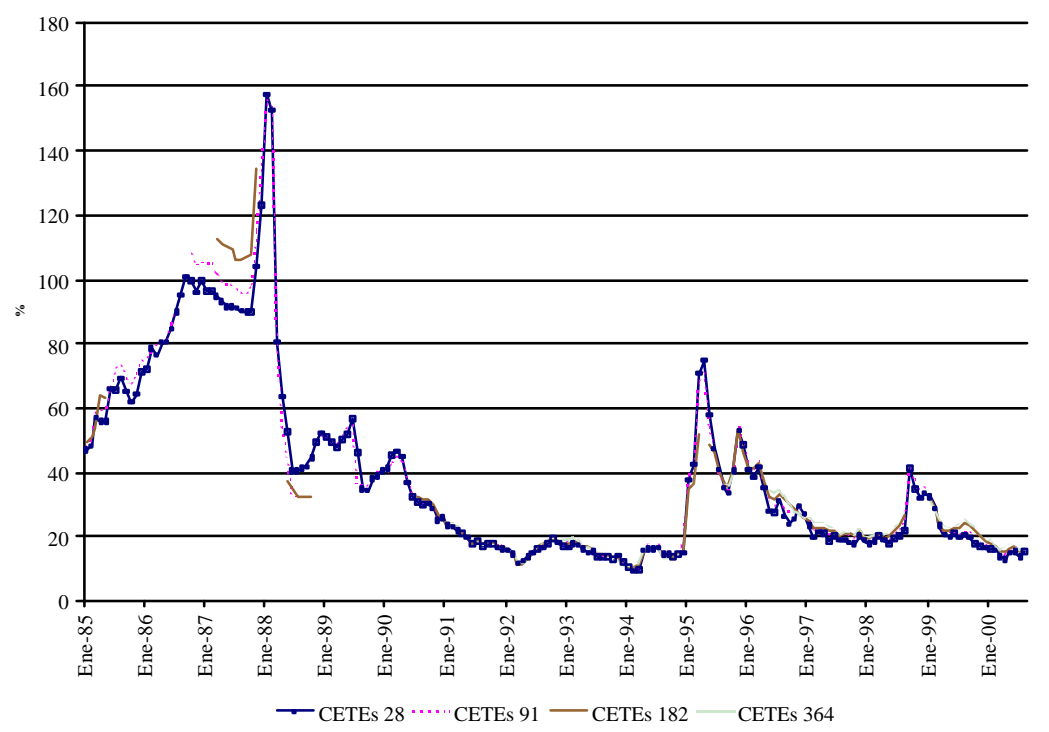

Fuente: Indicadores Bursátiles, Bolsa Mexicana de Valores.

Para poder llevar a cabo las pruebas empíricas que se describen más adelante mediante las técnicas usuales de regresión, es necesario que las series de tiempo consideradas sean estacionarias. En el Cuadro 1 se presentan las pruebas de Dickey-Fuller aumentada y de Phillips-Perron correspondientes a los diferenciales de las tasas de interés. Excepto para el diferencial $i_{6,3}$, la hipótesis nula de existencia de raíces unitarias es rechazada. El rechazo de la presencia de raíces unitarias en las variables asegura que los resultados de estimación no provengan de relaciones espurias entre las variables y que, por tanto, no sea necesario tomar diferencias de las variables definidas en las estimaciones. Estas dos propiedades sugieren la existencia de cointegración, ${ }^{10}$ es decir, de una combinación lineal estacionaria de largo plazo entre las tasas de interés, que por sí misma se considera una prueba débil de la HE. La razón de ello es que en ausencia de posibilidades de arbitraje, la diferencia de equilibrio entre las tasas de largo plazo y las de corto plazo es atribuible tan sólo a primas de riesgo o de liquidez de los diversos activos. Todas estas características concuerdan con las detectadas en Galindo (1995).

En el Cuadro 2 se presentan los resultados de las pruebas de causalidad de Granger entre los cambios de las tasas de interés y los diferenciales de las tasas. El no rechazo de la hipótesis

\footnotetext{
${ }^{10}$ Enders (1995).
} 
nula de que los diferenciales de las tasas de interés no causan en el sentido de Granger también constituye evidencia en favor de la HE. Esto se debe a que el diferencial de las tasas de interés en $t$ es el pronóstico óptimo sobre los cambios futuros de las tasas dado el conjunto de información disponible en ese periodo. Asimismo, el rechazo de la hipótesis de causalidad de la suma de los cambios en las tasas de interés en los diferenciales sugiere menos retroalimentación entre estas variables que la que encuentra Galindo (1995).

\section{Cuadro 1}

Pruebas de Raíz Unitaria de los Diferenciales de Tasas

\begin{tabular}{lcccc}
\hline & Niveles & \multicolumn{3}{c}{ Primeras } \\
& ADF & PP & PP \\
\hline$i_{3,1}$ & $*$ & $*$ & $*$ & $*$ \\
$i_{6,1}$ & $* *$ & $* *$ & $*$ & $*$ \\
$i_{6,3}$ & & & $*$ & $*$ \\
$i_{12,1}$ & $* *$ & $* *$ & $*$ & $*$ \\
$i_{12,6}$ & $*$ & $*$ & $*$ & $*$ \\
$\Delta_{3}$ & $*$ & $*$ & $*$ & $*$ \\
$\Delta_{6}$ & $*$ & $*$ & $*$ & $*$ \\
$\Delta_{12}$ & $*$ & $*$ & $*$ & $*$ \\
\hline y $^{* *}:$ Se rechaza la hipótesis de raíz unitaria al $1 \%$ y $5 \%$ &
\end{tabular}




\section{Cuadro 2}

Pruebas de Causalidad de Granger entre Diferenciales de Tasas de Interés y Sumas de Cambios en las Tasas de Interés

\begin{tabular}{lrrrrrrrr}
\hline & $\Delta_{3}$ & $\Delta_{6}$ & $\Delta_{12}$ & $i_{3,1}$ & $i_{6,1}$ & $i_{6,3}$ & $i_{12,1}$ & $i_{12,6}$ \\
$i_{3,1}$ & $*$ & $*$ & $\dagger$ & & & & & \\
$i_{6,1}$ & $*$ & $*$ & $*$ & & & & & \\
$i_{6,3}$ & $\dagger$ & $\dagger$ & $\dagger$ & & & & & \\
$i_{12,1}$ & $*$ & $*$ & $*$ & & & & & \\
$i_{12,6}$ & $*$ & $*$ & $*$ & & & & & \\
$\Delta_{3}$ & & & & $\dagger$ & $\dagger$ & $\dagger$ & $\dagger$ & $*$ \\
$\Delta_{6}$ & & & & $\dagger$ & $\dagger$ & $\dagger$ & $\dagger$ & $*$ \\
$\Delta_{12}$ & & & & $\dagger$ & $\dagger$ & $\dagger$ & $* *$ & $*$ \\
\hline
\end{tabular}

La hipótesis nula es: la variable en la columna no causa a la Granger a la variable en el renglón. Un asterisco indica que se rechaza la hipótesis nula al $1 \%$, dos asteriscos, se rechaza al $5 \%$. El signo $\dagger$ indica que no se rechaza la hipótesis nula

Concluida la descripción de los datos, a continuación se explican las dos principales pruebas empíricas de la HE seleccionadas para el presente análisis. Como se menciona en la sección previa, en la literatura de la ETTI se ha sugerido que el diferencial entre una tasa de interés de corto plazo y una tasa de largo plazo refleja una predicción sobre el comportamiento de la tasa de interés de corto plazo en el futuro. Una primera prueba de la HE de la ETTI se basa en su versión más simple, la cual postula que:

$$
R_{t}^{k} \approx \theta+\frac{1}{k} \sum_{i=0}^{k-1} E\left[r_{t+i}^{j}\right]
$$

en donde $R_{t}{ }^{k}$ es la tasa a $k$ periodos (tasa a largo plazo), $r_{t}^{j}$ es la tasa a $j$ periodos (tasa a corto plazo), $j=1$ en todos los casos y $\theta$ es una prima de riesgo constante. Esta expresión puede replantearse como:

$$
\sum_{i=0}^{k-1} E\left[r_{t+i}^{j}-r_{t}^{j}\right] \approx-k \theta+k\left(R_{t}{ }^{k}-r_{t}^{j}\right),
$$

Si los errores de pronóstico de las tasas de interés en $t$ están dados por:

$$
E r_{t+i}=r_{t+i}+v_{t+i}, \quad \forall i
$$

entonces, la ecuación (2) se convierte en:

$$
\Delta_{\mathrm{t}} \equiv \sum_{\mathrm{i}=0}^{k-1}\left[\mathrm{r}_{\mathrm{t}+\mathrm{i}}^{\mathrm{j}}-\mathrm{r}_{\mathrm{t}}^{\mathrm{j}}\right]=\alpha+\beta\left(\mathrm{R}_{\mathrm{t}}{ }^{\mathrm{k}}-\mathrm{r}_{\mathrm{t}}^{\mathrm{j}}\right)+\varepsilon_{\mathrm{t}}
$$


en donde $\varepsilon_{t}=\sum_{i=1}^{k-1} v_{t+i}, \alpha=-k / \boldsymbol{\theta}$ y $\boldsymbol{\beta}=k$. Esta teoría relaciona el cambio en la tasa de interés de corto plazo con el diferencial de las tasas de interés. Dicho diferencial refleja un pronóstico sobre el comportamiento futuro de las tasas de interés de corto plazo. Bajo el supuesto de que la información nueva no está correlacionada con la información actual, la prueba de esta teoría es equivalente a verificar si las expectativas son correctas (racionales) en promedio. ${ }^{11}$ Por otra parte, bajo la hipótesis nula, el término de error de la ecuación es ortogonal a los cambios en las tasas de interés de corto plazo. En consecuencia, la estimación de (4) mediante $M C O$ produce estimadores consistentes de los coeficientes. ${ }^{12}$ No obstante, debido a que los diferenciales de las tasas entre 1 y 3 meses o entre 3 y 6 meses se construyen, por ejemplo, con información mensual, es conveniente emplear la corrección de Newey-West para obtener errores estándar válidos en la regresión. ${ }^{13,14}$

La segunda prueba empírica seleccionada se fundamenta en la relación entre las tasas de interés forward y las tasas de interés spot. Según McCulloch y Shiller (1990), de todos los estudios sobre la hipótesis de expectativas racionales para la ETTI, los resultados de mayor

\footnotetext{
${ }^{11}$ Mankiw (1986) presenta una versión de la ecuación (4), basada en instrumentos de inversión que pagan un rendimiento de $\$ 1.00$ cada periodo. En dicha versión, el cambio inmediato de un periodo a otro de la tasa de interés de largo plazo es proporcional al diferencial de las tasas de largo y corto plazo en una magnitud igual a la tasa promedio de largo plazo. Esta prueba no es fundamentalmente distinta a la discutida aquí. Si la regresión del cambio de la tasa de largo plazo con respecto al diferencial de las tasas produce un coeficiente significativamente distinto al promedio de la tasa de largo plazo, entonces el diferencial produce un pronóstico equivocado con respecto al cambio de la tasa de largo plazo.

12 Si la distribución asintótica del coeficiente estimado $\hat{\beta}$ se concentra en un valor particular $\mathrm{k}$ al incrementar el tamaño de la muestra hasta el infinito, se dice que $k$ es la probabilidad límite de $\hat{\beta}$ y se escribe como plim $\hat{\beta}=k$. Si $\operatorname{plim} \hat{\beta}=\beta$, entonces se dice que $\hat{\beta}$ es consistente.

${ }^{13}$ Según Hansen y Hodrick (1980), esto se debe a que la construcción de variables explicativas a partir de datos con una periodicidad más frecuente que la de la variable que se des ea explicar mediante estimaciones de MCO produce autocorrelación en los errores de estimación. De ahí que éstos no tengan las propiedades asintóticas requeridas por MCO para hacer inferencia estadística. Por tanto, una estrategia de estimación adecuada y simple en términos computacionales consiste, precisamente, en realizar la estimación mediante MCO de los coeficientes de la regresión y el uso de errores estándar corregidos para que tengan las propiedades asintóticas apropiadas. Para una discusión más profunda sobre esta y otras estrategias de estimación apropiadas en estas circunstancias, véase Hansen (1979), Hansen y Hodrick (1980) y Newey y West (1987).

${ }^{14}$ La prueba que realizan González et al (1999) es una variante de las ecuaciones (2) y (3 ) en la cual:

$$
\Delta r_{t+1}=\alpha+\beta\left(R_{t}-r_{t}\right)+\varepsilon_{t},
$$

donde $\Delta r_{t+1}=r_{t+1}-r_{t}$ y la teoría de expectativas sugiere que $\beta$ debe ser mayor que cero. Sin embargo, Mankiw (1986) explica que esta implicación sólo es sugestiva y no se obtiene exactamente de la teoría debido a que las tasas de interés de corto plazo pueden no cambiar en el periodo siguiente, excepto cuando se considera la tasa de interés de largo plazo tiene vencimiento a 2 meses y la de corto plazo a 1 mes. Es por esta razón que a pesar de que algunos resultados de esta prueba con datos de México ofrecen cierta evidencia débil en favor de la validez de la hipótesis de expectativas racionales, ésta no se explora en el presente estudio.
} 
interés que se han obtenido son aquéllos en los que la variable explicativa es aproximadamente (o aproximadamente proporcional a) el diferencial entre una tasa forward del instrumento $i$ en el periodo $t$ aplicada entre los periodos $t+m$ y $t+m+n, \mathrm{f}_{\mathrm{t}, \mathrm{t}+\mathrm{m}+\mathrm{n}, \mathrm{t}+\mathrm{n}}$, y una tasa spot del instrumento $i$ en el periodo $t$ con el mismo vencimiento de la tasa forward, $r_{t, t+m}^{i}$. Este diferencial pronostica el cambio en la tasa spot $r_{t, t+m}^{i}$ durante los siguientes $n$ periodos. Las regresiones de los cambios en las tasas de interés de $m$ periodos con respecto a cambios predichos por la ETTI se expresan en la siguiente forma:

$$
r_{t+n, t+m+n}^{i}-r_{t, t+m}^{i}=\alpha+\beta\left(f_{t, t+m+n, t+n}^{i}-r_{t, t+m}^{i}\right)+\varepsilon_{t}
$$

en donde las tasas forward se construyen como

$$
f_{t, t+m+n, t+n}^{i}=\frac{m r_{m}-n r_{n}}{m-n}
$$

Aunque la evidencia de que $\beta>0$ significa que la tasa forward observada en $t$ tiene poder para predecir la tasa spot que se observará en $t+m+n$, lo cual Gonzále z et al (1999) reportan que se cumple para México, cabe enfatizar que la hipótesis nula asociada a la hipótesis pura de expectativas en esta regresión es $\beta=1$. Según Fama (1984), la ecuación (5) y la ecuación (5’)

$$
P \tau_{t+n}=\delta+\phi\left(f_{t, t+m+n, t+n}^{i}-r_{t, t+m}^{i}\right)+\eta
$$

donde $P \tau_{t+n}^{i}$ es el premio al vencimiento del bono con vencimiento $n$, definido como la diferencia entre el rendimiento mensual de $t$ a $t+1$ de un bono vigente $\tau$ meses hasta su vencimiento en $t$ y su precio en $t+1$, constituyen un sistema de ecuaciones con el que puede verificarse si el diferencial de la tasa forward contiene información para predecir la tasa spot futura o el premio al riesgo futuro. Como en la hipótesis pura de expectativas no hay premios al vencimiento en las tasas forward, toda la variación en el diferencial forward y spot se debe al cambio esperado en la tasa spot, $\beta=1$ y $\phi=0$. De manera alternativa, cuando toda la variación en la tasa forward se debe al premio al vencimiento esperado $\phi=1$ y $\beta=0$. 


\section{Resultados}

En esta sección se presentan las estimaciones de las pruebas sugeridas por las ecuaciones (4) y (5) descritas en la sección 3, se comparan e interpretan los resultados y, en cada caso, se presentan algunas pruebas adicionales propuestas en la literatura para ese fin.

Primeramente se estima la ecuación (4) utilizando datos de CETES con plazos 1, 3, 6 y 12 meses (es decir, $k \in\{3,6,12\}$ para el periodo de enero de 1985 a diciembre de 2000 y para las siguientes muestras: enero 1985-diciembre 1987; enero 1988-diciembre 1994 y enero 1996-diciembre 2000. Esto último se hace debido a la posibilidad de que el proceso de liberalización financiera o la adopción del régimen cambiario de tipo de cambio flexible hubiesen inducido variaciones en las medias condicionales estimadas en las regresiones. Los resultados se presentan en el siguiente cuadro: 


\section{Cuadro 3. Estimación de MCO de la Ecuación (4)}

\begin{tabular}{|c|c|c|c|c|}
\hline & $E_{+2}$ & & $E_{+6}$ & $E_{+12}$ \\
\hline & \multicolumn{4}{|c|}{$1986-2000$} \\
\hline$\alpha$ & -0.197 & & -0.832 & 6.265 \\
\hline$\beta$ & 0.054 & & 0.043 & 0.147 \\
\hline$R^{2}$ & 0.012 & & 0.002 & -0.002 \\
\hline \multirow[t]{2}{*}{ Obs } & 718 & & 671 & 524 \\
\hline & \multicolumn{4}{|c|}{$1985-1988$} \\
\hline$\alpha$ & 1.348 & & 3.353 & \\
\hline$\beta$ & -0.013 & & -0.016 & \\
\hline$R^{2}$ & -0.001 & & -0.007 & \\
\hline \multirow[t]{2}{*}{ Obs } & 98 & & 93 & \\
\hline & \multicolumn{4}{|c|}{ 1988-1994 } \\
\hline$\alpha$ & -0.790 & & -0.232 & -2.070 \\
\hline$\beta$ & 0.328 & 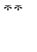 & 0.099 & 7.557 \\
\hline$\overline{R^{2}}$ & 0.100 & & 0.014 & 0.071 \\
\hline \multirow[t]{2}{*}{ Obs } & 359 & & 330 & 162 \\
\hline & \multicolumn{4}{|c|}{$1996-2000$} \\
\hline$\alpha$ & -0.533 & & -1.534 & -10.403 \\
\hline$\beta$ & 0.095 & 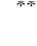 & 0.341 & 2.835 \\
\hline$R^{2}$ & 0.006 & & 0.065 & 0.113 \\
\hline Obs & 209 & & 196 & 169 \\
\hline
\end{tabular}

La comparación entre los resultados presentados en los páneles del Cuadro 3 sugiere que, efectivamente, los coeficientes varían entre los diferentes periodos. Pero para efectos de la hipótesis que se investiga, los aspectos más importante a destacar son que, en general, los coeficientes estimados difieren significativamente de los que predice la hipótesis de expectativas racionales de la ETTI, de acuerdo con h ecuación (4), y que éstos no son significativamente distintos de cero a los niveles de significancia convencionales. Cabe observar, en particular, que los coeficientes son menores que los que plantea la teoría (inclusive negativos para el periodo de 1985-1988), lo cual es indicativo de que los cambios de las tasas de interés son menores que los que sugiere el diferencial. Un ejemplo resulta útil para entender las implicaciones prácticas que puede tener este resultado, más allá de si se cumple o no la HE. Supongamos que a un inversionista interesado en adquirir instrumentos de cobertura para protegerse de los posibles cambios en la tasa de interés de los CETES a 28 días, un vendedor de opciones le ofrece tal cobertura pagando una prima "actuarialmente justa" determinada según la ecuación (4) y, en consecuencia, el inversionista tiene que decidir entre si comprarla o no. Si el inversionista en cuestión realiza el análisis arriba presentado, su 
decisión correcta sería no comprar esa cobertura porque la ecuación sobreestima los cambios en esa tasa de interés y, por ende, la prima que le ofrecen es muy cara.

Por otra parte, cabe señalar que la no significancia estadística de los diferenciales y las bajas $\mathrm{R}^{2}$ obtenidas indican que, contrario a lo que plantea la HE, éstos parecen no contener información sobre la trayectoria que seguirán las tasas de interés de corto plazo. En realidad, Mankiw y Miron (1986) reportan que el rechazo de la prueba anterior con datos de tasas de interés de plazos menores que un año es un hallazgo común en los estudios que se han realizado para los Estados Unidos y que diferentes niveles de predicción corresponden a diferentes regímenes monetarios. Una posibilidad para explicar esos resultados sugerida por dichos autores es que en adición al carácter aleatorio de las tasas de interés de corto plazo, las tasas de largo plazo se mueven más que las de corto plazo debido a variaciones en el premio que se ofrece al vencimiento. ${ }^{15}$

La otra posibilidad que sugiere Mankiw y Miron (1986) es, simpkmente, que los cambios de la tasa de interés de corto plazo sean impredecibles porque la tasa de interés futura siempre es igual a la tasa de interés presente, lo cual implicaría que el diferencial entre las tasas de interés de corto y de largo plazo es constante y que, en consecuencia, los errores estándar de los coeficientes de la ecuación (4) son infinitos. ${ }^{\mathbf{1 6}}$ La versión más débil de esta explicación es que los cambios esperados de la tasa de interés de corto plazo sean cero, debido a una política de estabilización o de fijación de las tasas de interés por parte del banco central. Para investigar si los cambios esperados de las tasas de interés, $\Delta_{k}$, pueden ser pronosticados con información contemporánea, siguiendo a Mankiw [1986] se estima esta variable utilizando los rezagos de la misma como variable independiente. Como lo muestran los resultados de estas estimaciones, que se presentan en el apéndice, tanto en la muestra completa como en las

\footnotetext{
${ }^{15}$ Esta es una caracterización compatible con un régimen de tipo de cambio fijo o de banda deslizante como el vigente en México hasta diciembre de 1994. No obstante, ni la trayectoria de las tasas presentada en la Gráfica 1 ni los resultados de las pruebas de estacionariedad del Cuadro 1 apoyan la conclusión enunciada.

16 Para comprender mejor este argumento, cabe señalar que surge una relación aproximadamente lineal entre las variables independientes de la ecuación (4) cuando $r_{t+l}=r_{t}$. Esto se debe a que el diferencial entre las tasas de interés de largo y de corto plazo se colapsa en la prima de riesgo asociada a la tasa de interés de largo plazo (supuesta constante) cuando $E_{t} r_{t+l}=r_{t^{*} l}$, por expectativas racionales, en la ecuación (1). Esto produce multicolinealidad, cuya consecuencia indeseable es una gran varianza de los estimadores de MCO de la ecuación (4). Según Kennedy (1992), “(uno) de los supuestos del modelo clásico de regresión lineal es la no existencia de relaciones lineales exactas entre las variables independientes y la existencia de al menos tantas observaciones como variables independientes. Si se viola cualquiera de las partes de este supuesto, es imposible mecánicamente computar estimaciones de MCO; en efecto, el procedimiento de estimación simplemente se colapsa por razones matemáticas, justo como si alguien tratara de dividir entre cero."
} 
distintas submuestras, los cambios esperados de las tasas de interés sí pueden ser pronosticados. Por tanto, estos resultados sugieren descartar la segunda posibilidad.

Concluida la discusión sobre la prueba anterior, los resultados de las estimaciones de la ecuación (5) se muestran en el Cuadro 4:

Cuadro 4. Estimación de MCO de la Ecuación (5)

\begin{tabular}{|c|c|c|c|c|c|c|c|c|}
\hline & \multicolumn{2}{|c|}{$1986-2000$} & \multicolumn{2}{|c|}{ 1986-1988 } & \multicolumn{2}{|c|}{ 1988-1994 } & \multicolumn{2}{|c|}{$1996-2000$} \\
\hline & 3 meses & 6 meses & 3 meses & 6 meses & 3 meses & 6 meses & 3 meses & 6 meses \\
\hline$\alpha$ & 2.58 & -1.95 & -32.07 & -1.95 & 0.20 & -2.60 & -0.24 & -0.09 \\
\hline$\beta$ & $0.16^{\dagger}$ & $0.25^{*, \dagger}$ & $0.02 *$ & $0.25^{*, \dagger}$ & $0.01 \dagger$ & $0.45^{* *, \dagger}$ & $0.62 *$ & $0.23^{*, \dagger}$ \\
\hline$\overline{R^{2}}$ & 0.79 & 0.88 & $\overline{0.95}$ & 0.88 & 0.83 & $\overline{0.93}$ & $\overline{0.84}$ & 0.90 \\
\hline Obs & 435 & 241 & 244 & 241 & 242 & 114 & 154 & 120 \\
\hline
\end{tabular}

Nuevamente, el rechazo de h hipótesis nula $\beta=1$ sugiere que la hipótesis de expectativas racionales no se cumple. Obsérvese que este coeficiente resulta cercano a cero, lo cual es congruente con la variabilidad del premio al vencimiento sugerida por Fama (1984) y detectada también en la prueba anterior. Por tanto, el inversionista del ejemplo anterior nuevamente debería rechazar una prima basada en la ecuación (5). Sin embargo, obsérvese que el signo positivo asociado a $\beta$ en ambas pruebas sugiere que una ETTI más inclinada puede interpretarse como una señal de posibles incrementos futuros en las tasas de interés spot.

El resultado más frecuente en la literatura relativa a las estimaciones de esta prueba también es su rechazo. Esto, en razón de que el coeficiente de la pendiente resultante frecuentemente es mucho menor que 1 y a veces hasta negativo. Para el caso de los Estados Unidos, la revisión de McCulloch y Schiller (1990) indica que cuando $n$ y $m$ son pequeños, ambos menores que 1 año, el coeficiente de la pendiente resulta positivo (el signo correcto) pero sustancialmente menor que uno. A su vez, cuando $n$ es pequeño (menor que 1 año) y $m$ es grande (20 años o más) el diferencial entre la tasa forward y la tasa spot predice un cambio en las tasas de interés en la dirección equivocada. La sabiduría popular sugiere que la esencia de la hipótesis de expectativas racionales es que un diferencial descomunalmente amplio entre las tasas forward y las spot presentes anticipa un incremento de las tasas de interés, no una reducción, como indican los resultados de prácticamente todos los estudios realizados al 
respecto. En consecuencia, ha habido algunos intentos por descomponer esta diferencia con respecto al valor de 1 del coeficiente de la pendiente, atribuyendo una parte a errores en las expectativas y la otra a la existencia de un premio al riesgo cambiante. ${ }^{17}$ La aplicación empírica de esta metodología requiere información proveniente de encuestas de expectativas para medir los errores en las expectativas que no está disponible para todo el periodo de análisis. ${ }^{18}$ Por ende, ese ejercicio queda pendiente para un estudio futuro. Así, el resto del estudio presente se enfoca a investigar la posibilidad de un premio al riesgo cambiante (lo cual también recibe apoyo de los resultados de las estimaciones del cuadro A.1 del apéndice).

Pruebas de otro tipo para la hipótesis de expectativas racionales se apoyan en la idea de verificar si el premio al riesgo puede predecirse con una regresión simple que incluya valores corrientes y un rezago del dferencial de las tasas. Esto se debe a que la constancia del diferencial de las tasas con respecto al plazo (es decir, un premio al vencimiento o al riesgo constante), significa en última instancia poder pronosticar un exceso de rendimiento o diferencias entre las tasas de interés spot y forward mediante información disponible en $t$. Dicha proposición puede apreciarse más claramente definiendo el premio al riesgo a partir de las tasas de interés spoty forward. Así, la ecuación puede rescribirse para obtener el premio al riesgo de la siguiente manera:

$$
y_{t} \equiv\left(1+R_{t}\right)^{k}-\sum_{i=0}^{k-1}\left[1+r_{t+i}\right] \approx k R_{t}-\sum_{i=0}^{k-1} r_{t+i}
$$

De acuerdo con esta expresión, el premio al riesgo, $y_{t}$, es igual a la diferencia esperada entre el rendimiento del bono de largo plazo y el del bono de corto plazo. Por tanto, esta expresión permite probar la $E H$ de manera similar a la ecuación (4).

Como se menciona en la sección previa, la teoría de expectativas racionales postula que el premio al riesgo es constante a través del tiempo. Si las expectativas son racionales, el premio al riesgo no puede ser predicho con variables previamente conocidas. Por tanto, en la regresión

\footnotetext{
${ }^{17}$ Froot (1987) encuentra que para los pronósticos a 3 meses adelante basados en las tasas de tres meses esta desviación del valor de 1 se explica principalmente por un premio al riego cambiante. Por su parte, en el caso de los pronósticos de cambios en las tasas de interés hipotecarias a 30 años casi toda la desviación se debe a errores de expectativas.

18 A partir de 1997 el Banco de México empezó a levantar una encuesta de expectativas económicas entre especialistas financieros cuya información puede emplearse para ese fin.
} 


$$
y_{t}=\alpha+\beta x_{t}+\varepsilon_{t}
$$

en donde $x_{t}$ es un vector de variables conocidas en $t, \varepsilon_{t}$ es el error de pronóstico con media cero y varianza constante, la hipótesis nula es $\beta=0$.

Según Mankiw (1986) a continuación se estiman dos versiones de la ecuación (7), una incluyendo como variable explicativa el diferencial de las tasas en $t$ y en $t-1$ y otra incluyendo rezagos del premio al riesgo. Los resultados de este ejercicio, presentados en el Cuadro 5, también permiten rechazar la hipótesis nula. Ello, al obtenerse coeficientes significativamente distintos de cero asociados a las variables explicativas prácticamente en todas las muestras (aunque no al considerarse el periodo completo). Puesto de otra manera, el premio al riesgo puede predecirse con valores históricos de las variables empleadas. Pero además, los rezagos del propio premio explican una mayor proporción del premio al riesgo que los rezagos del diferencial de las tasas, según lo indican los valores de la $R^{2}$ Ajustada.

Cuadro 5. Estimación de la Ecuación (7)

\begin{tabular}{|c|c|c|c|c|c|c|c|c|c|c|c|c|c|c|c|}
\hline \multicolumn{16}{|c|}{ Panel A: Variable explicativa: diferencial de tasas } \\
\hline & $Y_{3}$ & $* *$ & $Y_{6}$ & $Y_{3}$ & $Y_{6}$ & $Y_{12}$ & $Y^{3}$ & * $Y_{6}$ & & $Y_{12}$ & * & $Y_{3}$ & & $Y_{6}$ & $Y_{12}$ \\
\hline $\mathrm{C}$ & -2.13 & $*$ & 109.81 & 0.56 & 19.79 & 16.47 & 2.14 & 31.16 & & 50.29 &  & 3.19 & & 21.20 & 21.47 \\
\hline$I^{k, 1}(t)$ & 3.18 & $*$ & 5.06 & 1.80 & 4.45 & 4.46 & 1.68 & 3.26 & *** & 9.55 & 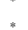 & -0.01 & & 8.87 & 10.28 \\
\hline $\mathrm{R}$ & 0.76 & & 0.59 & 0.49 & 0.41 & 0.03 & 0.17 & 0.13 & & 0.14 & & 0.00 & & 0.79 & 0.16 \\
\hline Obs & 84 & & 27 & 346 & 248 & 133 & 205 & 160 & & 153 & & 621 & & 447 & 294 \\
\hline \multicolumn{16}{|c|}{ Panel B: Variable explicativa: premio al riesgo } \\
\hline $\mathrm{Yk}^{\mathrm{k}}(\mathrm{t}-2)$ & -0.12 & & 0.08 & 0.04 & 0.01 & 0.00 & -0.21 & 0.10 & & 0.00 & & -0.04 & & -0.01 & -0.07 \\
\hline $\mathrm{Y}_{2}^{\mathrm{k}}(\mathrm{t}-3)$ & -0.05 & & 0.02 & -0.09 & -0.10 & -0.28 & 0.15 & -0.10 & & -0.10 & & 0.06 & & -0.07 & -0.15 \\
\hline $\mathrm{R}^{2}$ & 0.87 & & 0.68 & 0.48 & 0.70 & 0.89 & 0.31 & 0.82 & & 0.90 & & 0.60 & & 0.96 & 0.94 \\
\hline مhs & -77 & & 23 & 332 & 234 & 118 & 196 & 154 & & 149 & & 596 & & 417 & 273 \\
\hline
\end{tabular}

Para verificar si estos resultados se modifican al considerar la existencia de un premio al riesgo fluctuante, siguiendo a Sod (1997) se estima un modelo $\mathrm{GARCH}^{19}$ simple, en el cual la media condicional del modelo se caracteriza de acuerdo con las variantes de la ecuación (7) presentadas y la varianza del término de error se caracteriza de acuerdo con:

$$
h_{t}=\alpha_{0}+\alpha_{1} \varepsilon_{t-1}^{2}+\varphi h_{t-1}
$$

En esta expresión $h_{t}$ sigue un proceso $\operatorname{GARCH}(1,1)$, es decir, un proceso que depende del término de error al cuadrado del periodo anterior (efecto $\mathrm{ARCH}), \varepsilon_{t-1}^{2}, \mathrm{y}$ de la varianza del periodo anterior (efecto GARCH), $h_{t-1}$. También se estimó una versión del GARCH(1,1) en el

\footnotetext{
${ }^{19}$ Del inglés: Generalized Autorregresive Conditional Heteroskedastic Model.
} 
cual la media condicional simplemente es igual a una constante más un error y, para las tres especificaciones, se permite que los efectos GARCH entren en la regresión de la media condicional como un modelo GARCH-M. ${ }^{20,21}$ Hay dos ventajas importantes con respecto al ejercicio de Sod (1997) que a pesar de todo deben resaltarse. La primera es que en este estudio se emplea información más desagregada (semanal en vez de mensual o trimestral), que hace el uso de tales métodos más apropiado. ${ }^{22}$ La segunda es que, como en las estimaciones previas, el mayor número de observaciones a este nivel de agregación aunado a un mayor número de años, permite estimar por separado las muestras correspondientes a diferentes periodos. Esto último ha mostrado en las demás estimaciones que se obtienen diferencias importantes en los coeficientes estimados según el periodo considerado. Los resultados correspondientes se presentan a continuación. ${ }^{23}$

${ }^{20}$ Del inglés: Generalized Autorregresive Conditional Heteroskedastic-In-Means Model.

${ }^{21}$ Para la especificación en que la media condicional sólo depende de una constante y de un término de error, corresponde el siguiente GARCH-M:

$$
\begin{gathered}
y_{t}=c+\gamma h_{t}^{1 / 2}+\varepsilon_{t}, \\
h_{t}=\alpha_{0}+\alpha_{1} \varepsilon_{t-1}^{2}+\varphi h_{t-1}
\end{gathered}
$$

Enders (1995) aporta una explicación detallada de todos estos modelos.

\footnotetext{
${ }^{22}$ Esto simplemente se debe al hecho de que en la información agregada a nivel mensual o trimestral se diluyen en gran medida las fluctuaciones de la volatilidad, que son precisamente lo que se quiere captar a través de los modelos de tipo GARCH.

${ }^{23}$ En los cuadros hay algunos espacios vacíos en las celdas que corresponden a las estimaciones con variables de mayor plazo. Esto se debe a que en su construcción forzosamente se pierden algunas observaciones y, por tanto, el número disponible de ellas resulta insuficiente para realizar los cálculos.
} 
Cuadro 6. Estimación de los Modelos GARCH y GARCH-M

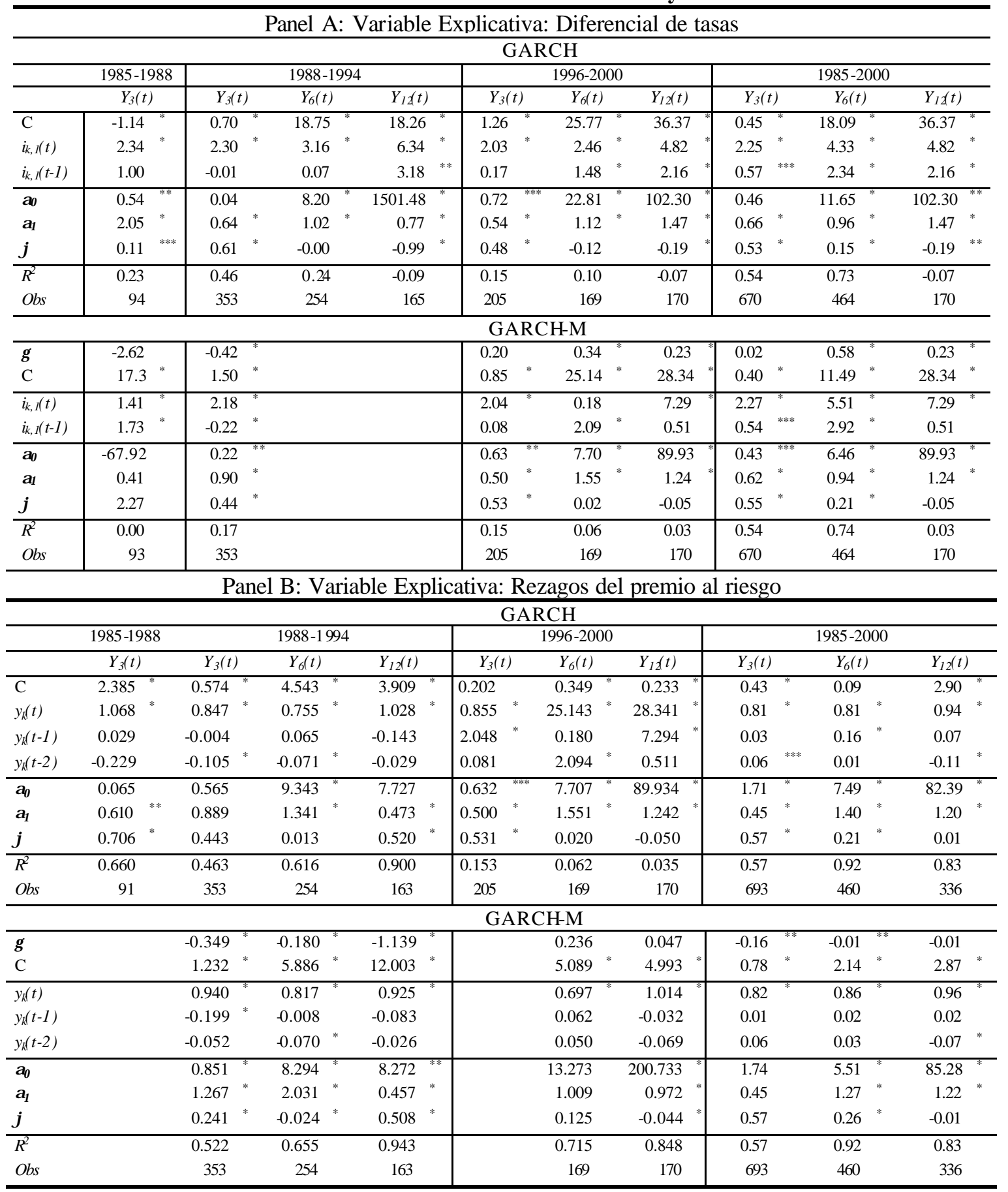

La presencia de efectos de heteroskedasticidad condicional se aprecia en los coeficientes significativamente distintos de cero asociados a los términos GARCH y $\mathrm{ARCH}$ en prácticamente todas las muestras. Esto coincide con los resultados obtenidos por Sod (1997). Pero, en contraste con dicho estudio, en el que aquí se reporta tanto los rezagos del diferencial 
de las tasas como del premio al riesgo siguen siendo útiles para pronosticar el premio, aún considerando la posibilidad de que fluctúe. Esto puede apreciarse tanto en el hecho de que la magnitud de los coeficientes de la regresión de la media condicional prácticamente no disminuye al incluirse tales efectos, como en el hecho de que las ecuaciones que especifican al premio como función de los rezagos exhiben mayores $R^{2}$ Ajustadas que aquéllos en los cuales la media se especifica como constante (en estos últimos inclusive se detectan casos en que el valor de este estadístico resulta negativo). Asimismo, las $R^{2}$ Ajustadas siguen siendo más bajas cuando se emplea el diferencial de las tasas que cuando se emplea al propio premio en términos rezagados.

Existen algunas razones para tomar los resultados anteriores con cierta cautela. La primera es que aunque la evidencia apunta hacia la existencia de un premio al riesgo que fluctúa en el tiempo, el hecho de que prácticamente en todas las ecuaciones la suma de los coeficientes GARCH y ARCH sea mayor que 1, denota que la varianza del proceso de error crece a través del tiempo. Esta es una razón para suponer que dicho proceso pudiera no ser una caracterización adecuada del riesgo. La segunda es que la inclusión de la varianza del error en la ecuación de la media condicional tampoco parece ser una caracterización adecuada de los datos anteriores a 1996. Esto se debe a que en todas las estimaciones de los modelos GARCH-M con las muestras anteriores a esa fecha el coeficiente asociado a esta variable resulta negativo, implicando que el premio al riesgo decrece al aumentar su propia volatilidad. Como es obvio, esto tampoco corresponde con la manera en que habitualmente se piensa sobre ese tema.

\section{Conclusiones}

Con el uso de una base de datos para México considerablemente más grande que la utilizada en estudios anteriores sobre el tema, en este trabajo se investiga si se cumple la $\mathrm{HE}$ en el caso de México. Los resultados obtenidos no permiten rechazar pr completo esta hipótesis, lo cual no resulta extraño a la luz de las conclusiones obtenidas en estudios realizados en otros países. Los resultados presentes concuerdan en señalar la impredecibilidad de las tasas de interés, debido probablemente a las fluctuaciones de las primas al riesgo en los diferenciales de las tasas de interés, como uno de los principales factores causales. Sin embargo, quedan 
pendientes para investigaciones futuras el examen de los posibles errores en la formación de las expectativas de los agentes. Un análisis de este tipo puede realizarse fácilmente debido a que recientemente se han comenzado a recabar encuestas entre especialistas financieros sobre los valores esperados de las diferentes variables económicas. Disponiendo de cotizaciones semanales de las diferentes tasas de interés, también podrían aplicarse métodos para modelar la volatilidad de las variables que requieren mayor desagregación de la información. Esto podría resultar en una mejor caracterización de las primas de riesgo que la que se obtiene mediante modelos GARCH.

Los hallazgos de este trabajo también se enmarcan dentro de los encontrados por sus autores sobre el contenido de información de expectativas captado en la ETTI. Los diferenciales de las tasas de interés parecen ser poco útiles para predecir los movimientos de las tasas de interés spot excepto de manera muy amplia, debido a su gran volatilidad. En consecuencia, no es sorprendente que muchas encuestas recabadas entre autoridades financieras de todo el mundo han revelado que la ETTI es sólo uno entre los muchos indicadores financieros y entre una gran diversidad de modelos y encuestas útiles para auxiliar la toma de decisiones en materia de política económica cotidianamente. Ello, pese a sus ventajas sobre otros mecanismos para la medición de expectativas que se derivan del hecho de que esta variable se puede obtener con gran oportunidad y que representa un consenso amplio del mercado. ${ }^{24}$

\footnotetext{
${ }^{24}$ Mylonas y Schich (1999) presenta los resultados de una encuesta reciente entre las autoridades monetarias de países miembros de la OCDE. Sus conclusiones señalan, precisamente, que para la evaluación de las condiciones monetarias y de las fuerzas económicas subyacentes más generales en la mayoría de los países encuestados se ha adoptado un enfoque ecléctico. Tal enfoque incluye la observación de varios indicadores de precios financieros que complementan los resultados de los modelos formales.
} 


\section{Bibliografía}

1) Campbell, J., R. Shiller y K. Schoenholtz, 1983, "Forward rates and future policy: interpreting the term structure of interest rates", Brookings Papers on Economic Activity 1, pp:173-217.

2) Castellanos, Sara, 2000, "El efecto del 'corto'sobre la estructura de tasas de interés", Documento de Investigación 2000-1, Banco de México, Enero.

3) Díaz de Leon, A. y L. Greenham, 2000, "Política Monetaria y Tasas de interés: Experiencia Reciente para el Caso de México", Documento de Investigación 2000-8, Banco de México, Diciembre.

4) DiNardo, J. y J. Johnston, 1997, Econometric Methods, 4ª ed., Mc Graw-Hill.

5) Enders, W., 1995, Applied Econometric Time Series, $1^{\text {st }}$ ed., John Wiley and Sons, Inc.

6) Estrella, A. y G. Hardouvelis, 1991, "The term structure as a predictor of real economic activity", The Journal of Finance 46, Junio, pp:555-76.

7) Fama, E. F., 1975, "Short-term interest rates as predictors of inflation", The American Economic Review 65, Junio, pp:269-82.

8) Fama, E. F., 1984, "The information in the term structure", Journal of Financial Economics 13, pp:509-28.

9) Fama, E. F., 1990,'Term-structure forecasts of interest rates, inflation and real returns", Journal of Monetary Economics 25, Enero, pp:59-76.

10) Froot, K. A., 1989, "New Hope for the Expectations Hypothesis of the Term Structure of Interest Rates", The Journal of Finance, Vol XLKV, No. 2, June, pp: 283-305.

11) Green, E., 1990, “Econometric Analysis”, McMillan.

12) Hamburger, M. y E. Platt, 1975, "The expectations hypothesis and the efficiency of the treasury bill market”, The Review of Economics and Statistics 57, Mayo: 190-99.

13) Hansen, L. P., 1979, "The Asymptotic Distribution of Least Squares Estimators with Endogenous Regressors and Dependent Residuals", Working Paper, Carnegie-Mellon Univ., Graduate School Indus. Admin., 1979.

14) Hansen, L.P. y R.J., Hodrick, 1980,'Forward exchange rates as optimal predictors of future spot rates: an econometric analysis" The Journal of Political Economy, 88, Octubre, pp:829-53.

15) Hardouvelis, G., 1988, "The predictive power of the term structure during recent monetary regimes", The Journal of Finance 43, Junio: 339-56.

16) Kennedy, P., 1992, “A Guide to Econometrics”, MIT Press, tercera edición. 
17) Mankiw, G., 1986(a), “The term structure of interest rates revisited", Brookings Papers on Economic Activity 1:61-110.

18) Mankiw, G. y J. Miron, 1986 "The changing khavior of the term structure of interest rates", The Quarterly Journal of Economics 101, Mayo:211-28.

19) Mishkin, F., 1990[a], "What does the term structure tell us about future inflation", Journal of Monetary Economics 25, pp:77-95.

20) Mishkin, F., 1990[b], "The information in the longer maturity term structure about future inflation", Quarterly Journal of Economics 105, Agosto, pp:815-28.

21) Mylonas, P. y Schich, S., 1999, "The Use of Financial Market Indicators by Monetary Authorities", Economics Department Working Papers No. 223, OECD.

22) Robertson, D., 1992, “Term structure forecasts of inflation”, The Economic Journal 102, Septiembre, pp: 1083-93.

23) Shiller, R., 1990, "The term structure of interest rates", en Friedman, B. y F. Hahn, Hanbook of Monetary Economics vol. I, pp:627-722.

24) Sod, G., 1996, "The term structure of interest rates: rationality, volatility and the Mexican experience (1982-1995)", tésis doctoral sin publicar, Harvard University. 


\section{Apéndice}

Cuadro A.1 Predicción de Cambios Futuros en las Tasas de Interés Mediante Rezagos

\begin{tabular}{|c|c|c|c|c|c|}
\hline & $\Delta_{k}(t-1)$ & $\Delta_{k}(t-1)$ & $\Delta_{k}(t-1)$ & $R^{2}$ & Obs \\
\hline & \multicolumn{5}{|c|}{ Muestra completa } \\
\hline$\Delta_{3}$ & -0.185 & 0.744 & $-0.230^{*}$ & 0.384 & 719 \\
\hline$\Delta_{6}$ & -0.216 & $1.054^{*}$ & $-0.232 *$ & 0.745 & 713 \\
\hline \multirow[t]{2}{*}{$\Delta_{12}$} & -0.357 & $1.212^{*}$ & $-0.289^{*}$ & 0.892 & 701 \\
\hline & \multicolumn{5}{|c|}{ 1986-1988 } \\
\hline$\Delta_{3}$ & -0.163 & 0.785 & -0.161 & 0.464 & 152 \\
\hline$\Delta_{6}$ & -0.299 & $1.118^{*}$ & $-0.258^{* *}$ & 0.801 & 152 \\
\hline \multirow[t]{2}{*}{$\Delta_{12}$} & -0.723 & $1.310^{*}$ & $-0.372^{*}$ & 0.922 & 152 \\
\hline & \multicolumn{5}{|c|}{ 1988-1994 } \\
\hline$\Delta_{3}$ & $-0.270 * *$ & 1.005 & $-0.319^{* * *}$ & 0.448 & 308 \\
\hline$\Delta_{6}$ & -0.230 & $1.107^{*}$ & $-0.309^{* *}$ & 0.691 & 303 \\
\hline \multirow[t]{2}{*}{$\Delta_{12}$} & 0.484 & $1.338^{*}$ & $-0.379^{*}$ & 0.886 & 297 \\
\hline & \multicolumn{5}{|c|}{$1996-2000$} \\
\hline$\Delta_{3}$ & 0.055 & 0.621 & -0.318 & 0.296 & 261 \\
\hline$\Delta_{6}$ & 0.043 & $0.969^{*}$ & $-0.223^{*}$ & 0.643 & 260 \\
\hline$\Delta_{12}$ & -0.595 & $1.073^{*}$ & $-0.191^{* * *}$ & 0.823 & 254 \\
\hline
\end{tabular}

